\title{
PCOS and risk of fractures
}

\author{
Yueh-Han $\mathrm{Hsu}^{1,2,3,4}$ (D) Hsin-Yi Yang ${ }^{1}$
}

Received: 13 February 2018 / Accepted: 14 February 2018 / Published online: 8 March 2018

(c) The Japanese Society for Bone and Mineral Research and Springer Japan KK, part of Springer Nature 2018

\section{Authors reply}

We are grateful for comments on our study [1] from Dr. Comim et al. They raised the important roles of BMI played in the risk of fracture in patients with polycystic ovary syndrome (PCOS), and mentioned the outcomes discrepancies in different literatures addressing similar topic. We agreed to the importance of BMI; yet, as reported in our study, there seemed different clinical characteristics of PCOS in different ethnic groups. For example, the prevalence of hirsutism and obesity in Chinese women with PCOS appears to be lower than that from Caucasian patients [2]. Besides, the average BMI in Taiwanese PCOS women was reported much lower than that in Western women [3]. As such, the impact of BMI might be lower in Chinese women with PCOS. However, none of the above statements may justify absence of BMI suppose we have access to the information. As we have stated in the limitation, the characteristics of our dataset lack certain important data including body weight and BMI.

The comment inquired about the incidence of lower leg fractures in our study. However, the fracture in lower extremity (knee, ankle, and foot) is not contained in the definition of osteoporotic and non-osteoporotic fractures in our study; therefore, our study did not analyze the fracture in lower extremity.

Best regards,

Yueh-Han Hsu MD, PhD

Hsin-Yi Yang PhD

\section{Compliance with ethical standards}

Conflict of interest The authors declared that there is no conflict of interest.

\section{References}

1. Yang HY, Lee HS, Huang WT, Chen MJ, Chen SC, Hsu YH (2017) Increased risk of fractures in patients with polycystic ovary syndrome: a nationwide population-based retrospective cohort study. J Bone Miner Metab. https://doi.org/10.1007/s0077 4-017-0894-8

2. Hsu MI, Liou TH, Chou SY, Chang CY, Hsu CS (2007) Diagnostic criteria for polycystic ovary syndrome in Taiwanese Chinese women: comparison between Rotterdam 2003 and NIH 1990. Fertil Steril 88:727-729

3. Zhao Y, Qiao J (2013) Ethnic differences in the phenotypic expression of polycystic ovary syndrome. Steroids 78:755-760

Yueh-Han Hsu

cych07023@gmail.com

1 Clinical Medical Research Center, Ditmanson Medical Foundation Chia-Yi Christian Hospital, Chia-Yi 600, Taiwan

2 Department of Medical Research, China Medical University Hospital, Taichung 404, Taiwan

3 Department of Internal Medicine, Ditmanson Medical Foundation Chia-Yi Christian Hospital, No. 539, Zhong-Xiao Road, Chia-Yi 600, Taiwan

4 Department of Nursing, Min-Hwei College of Health Care Management, Tainan 736, Taiwan 